\title{
Molecular Analysis and Chemical Evaluation of Ephedra Plants in Mongolia
}

\author{
Yuki Kitani, ${ }^{a}$ Shu Zhu, ${ }^{a}$ Takayuki Omote, ${ }^{a}$ Ken Tanaka, ${ }^{a}$ Javzan Batkhuu, ${ }^{b}$ Chinbat Sanchir, ${ }^{c}$ \\ Hirotoshi Fushimi, ${ }^{a}$ Masayuki Mikage, ${ }^{d}$ and Katsuko Komatsu* ${ }^{*}, a$ \\ ${ }^{a}$ Division of Pharmacognosy, Department of Medicinal Resources, Institute of Natural Medicine, University of Toyama; \\ 2630 Sugitani, Toyama 930-0194, Japan: ${ }^{b}$ Institute of Biology, National University of Mongolia; Ulaanbaatar-46, \\ Mongolia: ${ }^{c}$ Institute of Botany, Mongolian Academy of Science; Ulaanbaatar-17, Mongolia: and ${ }^{d}$ Graduate School of \\ Natural Science and Technology, Kanazawa University; Kakuma, Kanazawa, Ishikawa 920-1192, Japan. \\ Received October 14, 2008; accepted February 4, 2009; published online April 7, 2009
}

Ephedrae herba has been used in traditional Chinese and Japanese (Kampo) medicine from ancient times, with the primary resource being in China. In the present study, a field survey as well as molecular and chemical assessments were conducted on Ephedra plants in Mongolia to clarify whether they could be an alternative resource of the Ephedrae herba used in Japanese Kampo medicine. Ephedra sinica, E. equisetina, E. przewalskii, $E$. regeliana, $E$. monosperma and an unknown taxon (ESP) collected in Mongolia were divided into 9 genotypes on the basis of nucleotide sequences of $18 \mathrm{~S}$ ribosomal RNA (rRNA) gene and trnK gene. E. sinica, E. equisetina, and $E$. monosperma presented completely identical sequences to the corresponding species from China. The sequences of $\operatorname{trnK}$ gene and $18 \mathrm{~S}$ rRNA gene provide a useful index for identification and taxonomic classification of Mongolian Ephedra plants. Quantitative analysis of 5 ephedrine alkaloids revealed that almost all Mongolian Ephedra plants contained high amounts of total ephedrine alkaloids (TAs, $1.86-4.90 \%)$ and a high percentage of pseudoephedrine in TAs differed obviously from the Chinese. $E$. sinica and $E$. equisetina found in eastern and central Mongolia, showing total contents of ephedrine and pseudoephedrine higher than $1.43 \%$, were potential new resources of Japanese Pharmacopoeia grade Ephedrae herba.

Key words Ephedra; Mongolia; molecular identification; ephedrine alkaloid; HPLC analysis

Ephedrae herba, called "Mahuang" in Chinese and "Mao" in Japanese, has been used in traditional Chinese medicine and Kampo medicine for inducing perspiration, healing a cold, relieving cough and asthma and is included in a number of popular Kampo formulations such as Kakkonto and Shoseiryuto. In 1887, the main constituent, ephedrine, was first isolated by Dr. Nagayoshi Nagai and further developed as an effective medicine for asthma, opening a way to reaffirm the value and significance of traditional medicines. ${ }^{1)} \mathrm{Be}-$ sides ephedrine, other alkaloids such as pseudoephedrine, norephedrine and methylephedrine are also included in Ephedrae herba as active constituents. ${ }^{2)}$ Pharmacological studies revealed central nervous system (CNS) stimulation, anti-tussive, decongestion, anti-inflammatory activities of ephedrine and its analogue, ${ }^{3-6)}$ providing the scientific basis underlying its traditional uses. Besides traditional usage in Asia, it has also been used as a dietary supplement in U.S.A. and Europe in recent years. However, misuse or abuse of ephedrine-containing dietary supplement for the purpose of weight loss or euphoric stimulation has resulted in various adverse effects, such as headache, insomnia, heart palpitations, and even death. Therefore, U.S.A. and many western countries have imposed regulations or banned the sale of ephedrine-containing dietary supplements since 1994. ${ }^{7)}$

Ephedra plants are in high demand for both traditional medicinal use and also as raw materials for ephedrine extraction all over the world. In Japanese Pharmacopoeia (JP), Ephedrae herba is prescribed as the dried aerial parts of Ephedra sinica Stapf, E. intermedia Schrenk et C. A. Meyer and $E$. equisetina BunGE with no less than $0.7 \%$ of total alkaloids (the total of ephedrine and pseudoephedrine). In Japan, a high demand of about 500 tons/year of Ephedrae herba used in Kampo medicine has relied heavily on importation from China. ${ }^{8)}$ However, overharvesting has induced desertification and caused the Chinese government to curtail the exporting of Ephedra plants since 1999. ${ }^{9)}$ It is urgent to investigate the growth status in neighboring regions not only for new resources of Ephedrae herba but also for its protection and the development of further cultivation.

About 40 species belonging to the genus Ephedra are distributed in arid regions of east and north Africa, Asia, Europe, North America and South America, and 14 species in China. ${ }^{10)}$ In Mongolia, the northern neighbor of China, 9 Ephedra species are reported and 8 of them are common with those growing in China. ${ }^{11,12)}$ Therefore, we estimated that Mongolian Ephedra might be useful as an alternative resource of Ephedrae herba. Aiming to investigate the state of Ephedra plants in Mongolia, we carried out field surveys from 2002 to 2004, during which wide distribution of several Ephedra species was observed. Some populations in southern areas showed curved or twisted stems and other populations lacked clear characters for botanical classification. It is commonly known that Ephedra plants are quite difficult to identify because the morphological characters are very simple and easily change adapting to their growth environment. Molecular analysis and chemical fingerprinting methods ${ }^{13}$ ) have been used to identify Ephedra species, and molecular analyses of Chinese Ephedra plants based on chloroplast chlB gene, ${ }^{14)}$ trn $\mathrm{L} / \operatorname{trn} \mathrm{F}$ region and nuclear ribosomal DNA (rDNA) internal transcribed spacer 1 (ITS1), ITS2 sequence $^{15,16)}$ have been reported. No genetic investigation has been done, however, on Mongolian Ephedra plants. Our previous studies demonstrated that comparing sequences of nuclear $18 \mathrm{~S}$ rRNA gene and chloroplast $\operatorname{trnK}$ gene was a powerful tool to authenticate the botanical origin of herbal drugs and assist taxonomic classification. ${ }^{17,18)}$ Therefore, in the 


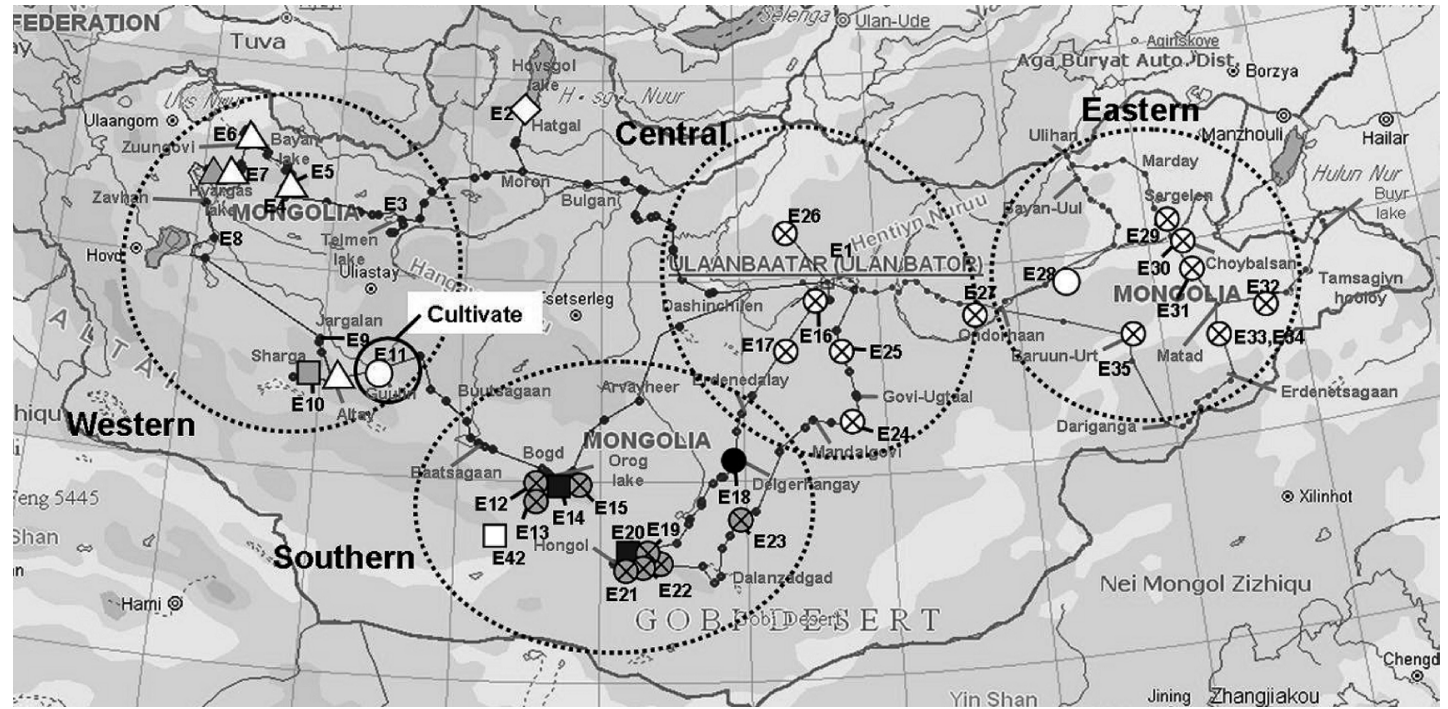

Fig. 1. Distribution Map of Ephedra Species and Their Genotypes in Mongolia

Collection points of Ephedra plants are indicated by "E No." Genotypes are shown by corresponding colors. E. equisetina and E. monosperma have the same genotype, thus the abbreviation of species name is added in parentheses. Marks indicate: white circle, genotype Si-I; cross in white circle, genotype Si-II; black circle, genotype E-I (E. equisetina); white diamond, genotype E-I (E. monosperma); cross in gray circle, genotype Esp; white box, genotype Pr-I; gray box, genotype Pr-II; black box, genotype Pr-III; white triangle, genotype Rg-I; gray triangle, genotype Rg-II.

Table 1. Plant Materials from China

\begin{tabular}{|c|c|c|c|c|c|c|}
\hline \multirow[b]{2}{*}{ Species } & \multirow[b]{2}{*}{ Voucher No. $\left.^{a}\right)$} & \multirow{2}{*}{$\begin{array}{l}\text { Geno } \\
\text { type }^{b)}\end{array}$} & \multirow[b]{2}{*}{ Locality } & \multirow{2}{*}{$\begin{array}{l}\text { Date of } \\
\text { collection }\end{array}$} & \multicolumn{2}{|c|}{ GenBank accession No. } \\
\hline & & & & & $\begin{array}{l}\text { 18S rRNA } \\
\text { gene }\end{array}$ & $\operatorname{trnK}$ gene \\
\hline Ephedra intermedia Schrenk. et C. A. MeYER & KANP02309 & In & Guinan, Qinghai Prov., China & 2002, Jul. 31 & & $\underline{\mathrm{AB} 453798}$ \\
\hline Ephedra intermedia Schrenk. et C. A. MeYer & KANP02363 & In & Yuzhong, Gansu Prov., China & 2002, Aug. 13 & AB453786 & \\
\hline Ephedra intermedia SCHRENK. et C. A. MeYer & KANP02369 & In & Longxi, Gansu Prov., China & 2002, Aug. 14 & & AB453/91 \\
\hline Ephedra sinica $\mathrm{STAPF}$ & KANP02109 & Si-II & E-er-duo-si, Inner Mongolia, China & 2002, Jun. 5 & & \\
\hline Ephedra sinica $\mathrm{STAPF}$ & KANP02133 & Si-II & Datong, Shanxi Prov., China & 2002, Jun. 8 & $\mathrm{AB}$ & AB453807 \\
\hline Ephedra przewalskii STAPF var. przewalskii & KANP02321 & Pr-I & Dulan, Qinghai Prov., China & 2002, Aug. 4 & & \\
\hline Ephedra przewalskii STAPF var. przewalskii & KANP02340 & Pr-I & $\begin{array}{l}\text { Xi-meng-gu-zu-zi-zhi-zhou, Qinghai } \\
\text { Prov., China }\end{array}$ & 2002, Aug. 7 & AB453790 & $\mathrm{AB} 453802$ \\
\hline Ephedra equisetina BUNGE & KANP02314 & E-I & Xining, Qinghai Prov., China & $2002, \mathrm{Al}$ & AB453784 & AB453795 \\
\hline Ephedra likiangensis FLoRIN & KANP0101001 & E-I & Kangding Xian, Sichuan Prov., China & 2001, Jul. 27 & AB453787 & AB453799 \\
\hline Ephedra monosperma GMeLIN ex C. A. Meyer & KANP02378 & E-I & Qilianshan, Qinghai Prov., China & 2002, Aug. 1 & AB453789 & AB453801 \\
\hline Ephedra gerardiana Waldich ex C. A. MeYer & KANP0101117 & E-I & Shiqu Xian, Sichuan Prov., China & 2001, Aug. 6 & AB453785 & AB453796 \\
\hline Ephedra minuta FLoRIN & KANP0101039 & $\mathrm{Mi}$ & Kangding Xian, Sichuan Prov., China & 2001, Jul. 28 & AB453788 & AB453800 \\
\hline
\end{tabular}

a) Voucher No. is specimen reference number of the Herbarium of the Medicinal Plants of the Faculty of Pharmaceutical Sciences in Kanazawa University. $b$ ) Genotype is determined on the basis of nucleotide sequences of $18 \mathrm{~S}$ rRNA gene and trnK gene.

present study, we determined the nucleotide sequences of these two regions and compared them with 8 clearly identified Chinese Ephedra plants for objective identification and to aid in the taxonomic resolution of Ephedra plants in Mongolia. In addition, clarification of the chemical equality of Mongolian Ephedra plants and the available Ephedrae herba is needed, because the constitutions of ephedrine alkaloids differ among species and there are also some species without these alkaloids. ${ }^{2}$ Subsequently, quantitative analysis on five major ephedrine alkaloids was conducted by HPLC- photodiode array detector (PDA) method for quality evaluation.

\section{MATERIALS AND METHODS}

Materials Twelve samples of 8 Ephedra species from China and 47 samples collected from Mongolia were ana- lyzed. The Chinese samples were identified by Prof. M. Mikage (Table 1). The vouchers are deposited in the Herbarium of Medicinal Plants, School of Pharmacy, College of Medical, Pharmaceutical and Health Science, Kanazawa University.

Mongolian samples were collected during our field investigations in 2002-2004 and 2006. Sample M874 was the only cultivated source in this study, which has been cultivated in Guulin, Govi-Altay Prov., Mongolia by using the seeds collected in Inner Mongolia, China; otherwise, all were wild plants distributed in Mongolia. Most of them were carefully identified by Dr. C. Sanchir in the Institute of Botany, Mongolian Academy of Science and the authors themselves as Ephedra sinica STAPF, E. equisetina BUnGe, E. monosperma Gmelin ex C. A. Meyer, E. regeliana Florin and E. przewalskii STAPF. However, specimens collected in southern Mongo- 


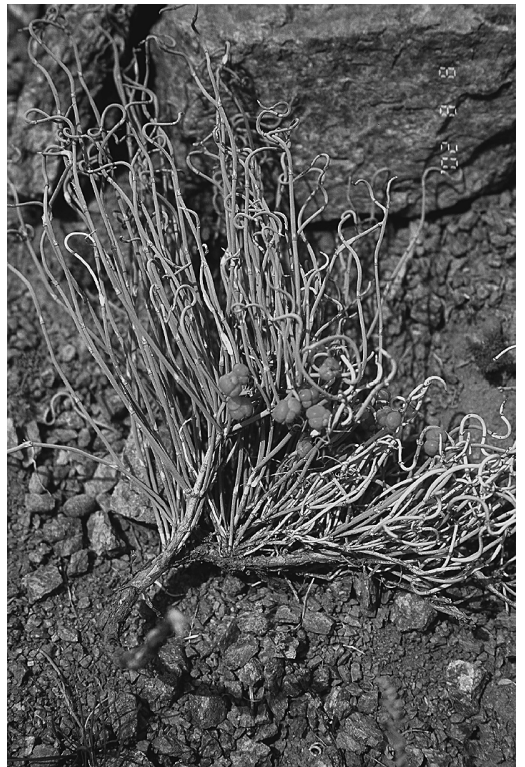

Fig. 2. Morphology of Specimen M929

Specimen M929 (genotype Esp) which was collected in Ih Bogdyn Nuruu, Bayanhongor Prov. shows a typically allopheric feature. The left part shows general morphology of ESP and the right part resembles E. przewalskii.

lia showed curved or twisted stems and some had allopheric features as shown in Fig. 2. Here, we tentatively treated these samples as Ephedra sp. (ESP) and included all of them in the following molecular and chemical analyses. It is noteworthy that due to fewer morphological characteristics being available in "Flora of Mongolia"11) and the "Key to the vascular plants of Mongolia,"12) floristic literature with valuable morphological information on Ephedra plants of neighboring regions were used as reference in morphological identification, such as "Flora Xinjiangensis,"19) "Flora of China" and "Flora of Siberia."

All the vouchers and samples from Mongolia are stored in the Museum of Materia Medica, Institute of Natural Medicine, University of Toyama, Japan (Table 2).

Isolation of Total DNA and PCR Amplification Total DNA was extracted from $50-100 \mathrm{mg}$ of dry stems by DNeasy Plant Mini Kit (Qiagen, Germany). Using it as template, 18S rRNA gene and two fragments with partial overlap of trnK gene (fragments A and B) regions were amplified by polymerase chain reaction (PCR), respectively (Figs. 3, 4). The PCR was performed using $10-100 \mathrm{ng}$ of total DNA as a template in $50 \mu \mathrm{l}$ of reaction mixture consisting of $1 \times \mathrm{GoTag}$ Flexi Buffer (pH 8.5, Promega, U.S.A.), $1.5 \mathrm{~mm} \mathrm{MgCl}_{2}$, $0.2 \mathrm{~mm}$ of each dNTP, 1.25 U Taq polymerase (Promega, U.S.A.), and $2.5 \mathrm{~mm}$ of each primer. Three pairs of primers were used for PCR amplification, that is, $18 \mathrm{~S} 5^{\prime} \mathrm{F}$ and ITS 57R for $18 \mathrm{~S}$ rRNA gene; trnK $3914 \mathrm{~F}$ and trnK In $3 \mathrm{R}$ for the fragment $\mathrm{A}$ of $\operatorname{trn} \mathrm{K}$ gene; $\operatorname{trnK}$ In $2 \mathrm{~F}$ and $\operatorname{trnK} 2 \mathrm{R}$ for the fragment B of trnK gene (Table 3). PCR amplifications were carried out in a Thermal Controller PTC-100 (MJ Research Inc., U.S.A.) with cycling conditions as shown below. $18 \mathrm{~S}$ rRNA gene: initial denaturation at $94{ }^{\circ} \mathrm{C}$ for $3 \mathrm{~min}$, followed by 35 cycles of denaturation at $94^{\circ} \mathrm{C}$ for $50 \mathrm{~s}$, annealing at $67^{\circ} \mathrm{C}$ for $50 \mathrm{~s}$, extension at $72^{\circ} \mathrm{C}$ for $2 \mathrm{~min}$, and then final extension at $72^{\circ} \mathrm{C}$ for $20 \mathrm{~min}$; fragment $\mathrm{A}$ of $\operatorname{trnK}$ gene: initial denaturation at $94^{\circ} \mathrm{C}$ for $3 \mathrm{~min}$, followed by 35 cycles of de- naturation at $94{ }^{\circ} \mathrm{C}$ for $50 \mathrm{~s}$, annealing at $56^{\circ} \mathrm{C}$ for $50 \mathrm{~s}$, extension at $72^{\circ} \mathrm{C}$ for $1 \mathrm{~min} 45 \mathrm{~s}$, and then final extension at $72{ }^{\circ} \mathrm{C}$ for $20 \mathrm{~min}$; fragment $\mathrm{B}$ of $\operatorname{trnK} \mathrm{K}$ gene: $94^{\circ} \mathrm{C}$ for $3 \mathrm{~min}$, 35 cycles of $94^{\circ} \mathrm{C}$ for $50 \mathrm{~s}, 54^{\circ} \mathrm{C}$ for $50 \mathrm{~s}, 72^{\circ} \mathrm{C}$ for $1 \mathrm{~min}$ $45 \mathrm{~s}$, and then $72^{\circ} \mathrm{C}$ for $20 \mathrm{~min}$. The $4 \mu \mathrm{l}$ of PCR product was resolved by $1.0 \%$ agarose gel electrophoresis and the remaining part was purified using a Montage PCR Centrifugal Filter Device (Millipore, U.S.A.).

Sequencing and Molecular Analysis Sequencing reaction of purified PCR products was carried out using ABI PRISM Bigdye Terminator v3.1 Cycle sequencing kits (Applied Biosystems, U.S.A.) with the primers shown in Table 3. Sequence was determined directly by an ABI Prism 3100Avant Genetic Analyzer (Applied Biosystems, U.S.A.). The analyzed sequences were assembled, and a consensus sequence of each sample was finally constructed. Then the obtained DNA sequences were aligned and compared by BioEdit program (ver. 7.0.8.2.).

HPLC Analysis of Ephedrine Alkaloids Quantitative analysis of five ephedrine alkaloids (ephedrine, pseudoephedrine, norephedrine, norpseudoephedrine and methylephedrine, Fig. 5) was conducted by the reported method ${ }^{21}$ ) with modification. It was performed by a Jasco HPLC system composed of a PU-1580 pump, a LC-1580-02 ternary gradient unit, and a MD-1510 multiwavelength detector. As for molecularly identified plants, the middle part of the stem except nodes was pulverized and then dried in a desiccator for $24 \mathrm{~h}$. An aliquot (ca. $150 \mathrm{mg}$ ) was extracted two times with $5 \mathrm{ml}$ of a mixed solvent of $\mathrm{H}_{2} \mathrm{O}$ : acetonitrile : phosphoric acid $=65: 35: 0.04$ with $0.4 \%$ sodium lauryl sulfate (SDS) by ultrasonic extraction for $30 \mathrm{~min}$ and centrifuged $3000 \boldsymbol{g}$ for $10 \mathrm{~min}$. The divided supernatants were combined and made up to a final volume of $10 \mathrm{ml}$. An aliquot of $10 \mu \mathrm{l}$ filtered through a $0.45 \mu \mathrm{m}$ filter was subjected to HPLC analysis under the following conditions: column, Waters Symmetry $\mathrm{C}_{18}$ column $(4.6 \mathrm{~mm} \times 150 \mathrm{~mm}$ i.d.); mobile phase, the same solvent as used in the extraction; flow rate, $0.8 \mathrm{ml} / \mathrm{min}$; column temperature, $30^{\circ} \mathrm{C}$. Detection was performed at $210 \mathrm{~nm}$.

Ephedrine and pseudoephedrine used as chemical standards were purchased from Daiichi Fine Chemical Co., Ltd. (Japan). Norephedrine was kindly provided by Dr. Yukiko Makino (Kanto-Shinetsu Regional Bureau of Health and Welfare, Japan). Methylephedrine standard was purified from methylephedrine parenteral solution (Fuso Pharmaceutical Industry, Ltd., Japan). Content of norpseudoephedrine was calculated using the calibration curve of norephedrine.

\section{RESULTS}

$18 S$ rRNA Gene and trnK Gene Sequences of Ephedra Plants from China The result of DNA analysis of Chinese Ephedra plants is shown in Table 4. The determined sequences of $18 \mathrm{~S}$ rRNA gene were $1792 \mathrm{bp}$ in length and 4 types of sequences were observed among the 8 species. $E$. sinica, E. intermedia and E. przewalskii possessed similar sequences with only 1 site difference. At nucleotide position 1709 from upstream, E. sinica presented double peaks of thymine (T)/cytosine (C) where the peak of $\mathrm{T}$ was obviously higher than that of $\mathrm{C}(\mathrm{T}>\mathrm{C})$, whereas $E$. intermedia possessed $\mathrm{T}$ and E. przewalskii had $\mathrm{C}$ at this position. The other 5 species (E. equisetina, E. likiangensis, E. monosperma, E. 
Table 2. Plant Materials Collected in Mongolia

\begin{tabular}{|c|c|c|c|c|c|c|c|c|}
\hline \multirow[b]{2}{*}{ Species } & \multirow{2}{*}{$\begin{array}{l}\text { Voucher } \\
\text { No. }{ }^{a)}\end{array}$} & \multirow[b]{2}{*}{ Genotype $^{b)}$} & \multirow[b]{2}{*}{ Point $^{c)}$} & \multirow[b]{2}{*}{ Locality } & \multirow[b]{2}{*}{$\begin{array}{l}\text { Altitude } \\
\text { (m) }\end{array}$} & \multirow{2}{*}{$\begin{array}{l}\text { Date of } \\
\text { collection }\end{array}$} & \multicolumn{2}{|c|}{ GenBank accession No. } \\
\hline & & & & & & & $\begin{array}{l}\text { 18S rRNA } \\
\text { gene }\end{array}$ & $\operatorname{trnK}$ gene \\
\hline Ephedra sinica & M874 & Si-I & E11 & $\begin{array}{l}\text { Guulin, Govi-Altay Prov. } \\
\text { (Cultivated; Seeds from Inner Mongolia) }\end{array}$ & 1829 & Aug. 5, 2002 & \multirow[t]{2}{*}{ AB453793 } & \multirow{16}{*}{ AB453807 } \\
\hline Ephedra sinica & M1157 & Si-I & E28 & Ondorhaan-Batnorov, Hentiy Prov. & 1060 & Jul. 14, 2004 & & \\
\hline Ephedra sinica & M969 & Si-II & E16 & Ulaanbaatar-Bayan-Onjuul, Tov Prov. & 1429 & Jul. 23, 2003 & \multirow{14}{*}{ AB453792 } & \\
\hline Ephedra sinica & M990 & Si-II & E17 & Bayan-Onjuul, Tov Prov. & 1437 & Jul. 24, 2003 & & \\
\hline Ephedra sinica & M1054 & Si-II & E24 & Ih Gazryn chuluu, Dundgovi Prov. & 1430 & Jul. 28, 2003 & & \\
\hline Ephedra sinica & M1056 & Si-II & E24 & Ih Gazryn chuluu, Dundgovi Prov. & 1430 & Jul. 28, 2003 & & \\
\hline Ephedra sinica & M1069 & Si-II & E25 & Mandalgovi-Ulaambaatar & 1508 & Jul. 28, 2003 & & \\
\hline Ephedra sinica & M1071 & Si-II & E26 & Bornuur-Bayangol, Selenge Prov. & 977 & Jul. 30, 2003 & & \\
\hline Ephedra sinica & M1156 & $\mathrm{Si}-\mathrm{II}$ & E27 & Jargalthaan-Moron, Hentiy Prov. & 1203 & Jul. 13, 2004 & & \\
\hline Ephedra sinica & M1210 & $\mathrm{Si}-\mathrm{II}$ & E29 & Sergelen-Choybalsan, Dornod Prov. & 828 & Jul. 17, 2004 & & \\
\hline Ephedra sinica & M1211 & Si-II & E30 & Choybalsan-Matad, Dornod Prov. & 836 & Jul. 18, 2004 & & \\
\hline Ephedra sinica & M1213 & Si-II & E31 & Choybalsan-Matad, Dornod Prov. & 831 & Jul. 18, 2004 & & \\
\hline Ephedra sinica & M1215 & $\mathrm{Si}-\mathrm{II}$ & E32 & $\begin{array}{l}\text { Sangiyn Dalaynuul-Tamsagiyn hooloy, } \\
\text { Dornod Prov. }\end{array}$ & 697 & Jul. 19, 2004 & & \\
\hline Ephedra sinica & M1227 & Si-II & E33 & Matad-Erdenetsagaan, Suhbaatar Prov. & 822 & Jul. 20, 2004 & & \\
\hline Ephedra sinica & M1229 & Si-II & E34 & Matad-Erdenetsagaan, Suhbaatar Prov. & 822 & Jul. 20, 2004 & & \\
\hline Ephedra sinica & M1242 & Si-II & E35 & Dariganga, Suhbaatar Prov. & 1058 & Jul. 21, 2004 & & \\
\hline Ephedra equisetina & M995 & E-I & E18 & Delgerhangay, Dundgovi Prov. & 1642 & Jul. 24, 2003 & AB453784 & \multirow{2}{*}{ AB453795 } \\
\hline Ephedra equisetina & M998 & E-I & E18 & Delgerhangay, Dundgovi Prov. & 1642 & Jul. 24, 2003 & $3 / 84$ & \\
\hline Ephedra monosperma & M581 & E-I & E2 & Hovsgol, Hovsgol Prov. & 1845 & Aug. 23, 2002 & AB453789 & $\mathrm{AB} 453801$ \\
\hline Ephedra regeliana & M707 & Rg-I & E4 & Songino-Jingiin halzan, Uvs Prov. & 1489 & Jul. 28, 2002 & \multirow{6}{*}{ AB453791 } & \multirow{5}{*}{ AB453805 } \\
\hline Ephedra regeliana & M778 & Rg-I & E6 & Bayannuur, Uvs Prov. & 983 & Jul. 28, 2002 & & \\
\hline Ephedra regeliana & M1301 & Rg-I & E7 & Talyn Har uul, Uvs Prov. & 1888 & Jul. 31, 2002 & & \\
\hline Ephedra regeliana & M1302 & Rg-I & E7 & Talyn Har uul, Uvs Prov. & 1888 & Jul. 31, 2002 & & \\
\hline Ephedra regeliana & M872 & Rg-I & E10 & Altay-Sharga, Govi-Altay, Prov. & 1498 & Aug. 4, 2002 & & \\
\hline Ephedra regeliana & M779 & Rg-II & E6 & Bayannuur, Uvs Prov. & 983 & Jul. 30, 2002 & & $\overline{\mathrm{AB} 453806}$ \\
\hline Ephedra przewalskii & M871 & Pr-II & E10 & Altay-Sharga, Govi-Altay, Prov. & 1498 & Aug. 4, 2002 & \multirow{5}{*}{ AB453790 } & $\underline{\mathrm{AB} 453803}$ \\
\hline Ephedra przewalskii & M931 & Pr-III & E14 & $\begin{array}{l}\text { Ih Bogdyn Nuruu-Baga Bogdyn Nuruu, } \\
\text { Bayanhongor Prov. }\end{array}$ & 1489 & Aug. 8, 2002 & & \multirow[t]{2}{*}{ AB453804 } \\
\hline Ephedra przewalskii & M1028 & Pr-III & E20 & Bulgan-Hongor, Omnogovi Prov. & 1618 & Jul. 25, 2003 & & \\
\hline Ephedra przewalskii & M1348-1 & Pr-I & E42 & Shinejinst-Ehiyn gol, Bayanhongor Prov. & 1460 & Jul. 25, 2006 & & \multirow{2}{*}{ AB453802 } \\
\hline Ephedra przewalskii & M1348-2 & Pr-I & E42 & Shinejinst-Ehiyn gol, Bayanhongor Prov. & 1460 & Jul. 25, 2006 & & \\
\hline Ephedra sp. & M928-1 & Esp & E13 & Ih Bogdyn Nuruu, Bayanhongor Prov. & 2186 & Aug. 8, 2002 & \multirow{19}{*}{ AB453794 } & \multirow{19}{*}{ AB453808 } \\
\hline Ephedra sp. & M928-2 & Esp & E13 & Ih Bogdyn Nuruu, Bayanhongor Prov. & 2186 & Aug. 8, 2002 & & \\
\hline Ephedra sp. & M928-3 & Esp & E13 & Ih Bogdyn Nuruu, Bayanhongor Prov. & 2186 & Aug. 8, 2002 & & \\
\hline Ephedra sp. & M929 & Esp & E13 & Ih Bogdyn Nuruu, Bayanhongor Prov. & 2186 & Aug. 8, 2002 & & \\
\hline Ephedra sp. & M930 & Esp & E13 & Ih Bogdyn Nuruu, Bayanhongor Prov. & 2186 & Aug. 8, 2002 & & \\
\hline Ephedra sp. & M950 & Esp & E15 & $\begin{array}{l}\text { Baruunbayan-Ulaan-Baga Bogdyn Nuruu, } \\
\text { Ovorhangay Prov. }\end{array}$ & 1961 & Aug. 9, 2002 & & \\
\hline Ephedra sp. & M951 & Esp & E15 & $\begin{array}{l}\text { Baruunbayan-Ulaan-Baga Bogdyn Nuruu, } \\
\text { Ovorhangay Prov. }\end{array}$ & 1961 & Aug. 9, 2002 & & \\
\hline Ephedra sp. & M952 & Esp & E15 & $\begin{array}{l}\text { Baruunbayan-Ulaan-Baga Bogdyn Nuruu, } \\
\text { Ovorhangay Prov. }\end{array}$ & 1961 & Aug. 9, 2002 & & \\
\hline Ephedra sp. & M953 & Esp & E15 & $\begin{array}{l}\text { Baruunbayan-Ulaan-Baga Bogdyn Nuruu, } \\
\text { Ovorhangay Prov. }\end{array}$ & 1961 & Aug. 9, 2002 & & \\
\hline Ephedra sp. & M954 & Esp & E15 & $\begin{array}{l}\text { Baruunbayan-Ulaan-Baga Bogdyn Nuruu, } \\
\text { Ovorhangay Prov. }\end{array}$ & 1961 & Aug. 9, 2002 & & \\
\hline Ephedra sp. & M1027-1 & Esp & E19 & Bulgan-Hongor, Omnogovi Prov. & 1471 & Jul. 25, 2003 & & \\
\hline Ephedra sp. & M1027-2 & Esp & E19 & Bulgan-Hongor, Omnogovi Prov. & 1471 & Jul. 25, 2003 & & \\
\hline Ephedra sp. & M1029 & Esp & E20 & Bulgan-Hongor, Omnogovi Prov. & 1618 & Jul. 25, 2003 & & \\
\hline Ephedra sp. & M1030 & Esp & E21 & Bulgan-Hongor, Omnogovi Prov. & 1783 & Jul. 25, 2003 & & \\
\hline Ephedra sp. & M1036 & Esp & E22 & Uliin Davaa, Omnogovi Prov. & 2035 & Jul. 26, 2003 & & \\
\hline Ephedra sp. & M1037 & Esp & E22 & Uliin Davaa, Omnogovi Prov. & 2035 & Jul. 26, 2003 & & \\
\hline Ephedra sp. & M1039 & Esp & E22 & Uliin Davaa, Omnogovi Prov. & 2035 & Jul. 26, 2003 & & \\
\hline Ephedra sp. & M1051 & Esp & E23 & Hanhongor-Huld, Omnogovi Prov. & 1264 & Jul. 27, 2003 & & \\
\hline Ephedra sp. & M1052 & Esp & E23 & Hanhongor-Huld, Omnogovi Prov. & 1264 & Jul. 27, 2003 & & \\
\hline
\end{tabular}

a) Voucher No. cited here is the abbreviation of Komatsu et al. M-No. b) Genotype is determined on the basis of nucleotide sequences of $18 \mathrm{~S}$ rRNA gene and trnK gene. c) Locality is shown in Fig. 1 with point number.

gerardiana and E. minuta) were of the same sequence which differed from that of E. sinica at nucleotide positions 625, 1381, 1683 and 1709.

The length of determined $\operatorname{trn} \mathrm{K}$ gene sequences ranged from 2307 to $2383 \mathrm{bp}$, and 13 sites of nucleotide differences were observed among the 8 species. E. intermedia had a long insertion consisting of 1 or 2 repeats of a $38 \mathrm{bp}$ sequence unit, which resulted in this sequence being the longest among 
the 8 Ephedra species. E. sinica, E. intermedia, E. przewalskii and E. minuta showed their own specific sequences in trnK gene and the remaining 4 species possessed the same sequence. Therefore, based on sequences of both genes, 8 Chinese Ephedra species were of 5 genotypes assigned as follows: Si-II type: E. sinica, In type: E. intermedia, Pr-I type: E. przewalskii, E-I type: E. equisetina, E. likiangensis, E. monosperma and E. gerardiana, Mi type: E. minuta (Table 4).

18S rRNA Gene and trnK Gene Sequences of Mongolian Ephedra Plants and Comparison with Those of Chinese Plants Ephedra plants collected in Mongolia were morphologically identified as 5 species (E. sinica, E. equisetina, E. monosperma, E. przewalskii and E. regeliana) and an unknown Ephedra sp. (ESP). Forty-nine samples were analyzed for sequences of $18 \mathrm{~S}$ rRNA gene and $\operatorname{trnK}$ gene in the same way as Chinese samples. The results divided Mongo-

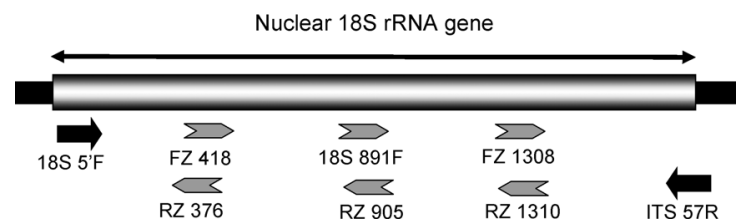

Fig. 3. Approach for Sequence Determination of 18S rRNA Gene

The positions of primers used in PCR amplification and sequence determination of $18 \mathrm{~S}$ rRNA gene are indicated by arrows and mountain-shaped arrows, respectively.

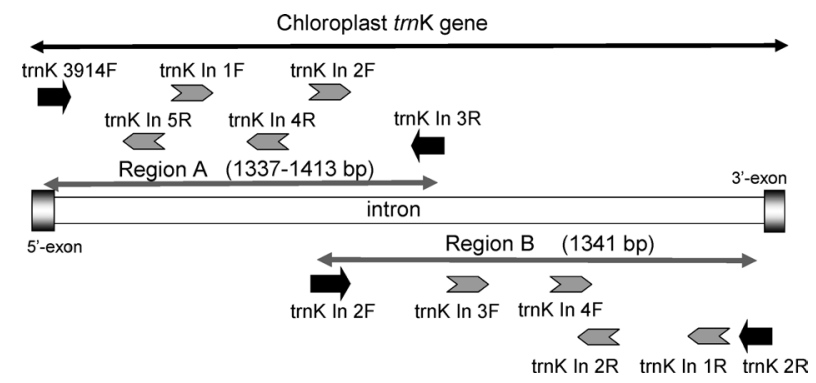

Fig. 4. Approach for Sequence Determination of trnK Gene

Two fragments with partial overlap were amplified by PCR to obtain the whole sequence of $\operatorname{trn\mathrm {K}}$ gene. The positions of primers used in PCR amplification and sequence determination of $\operatorname{trn\mathrm {K}}$ gene are indicated by arrows and mountain-shaped arrows, respectively.<smiles>CC(C)[C@H](O)c1ccccc1</smiles><smiles>C[C@H](N)[C@H](O)c1ccccc1</smiles>

pseudoephedrine<smiles>CC(N)C(O)c1ccccc1</smiles>

norephedrine<smiles>CC(N)C(O)c1ccccc1</smiles>

norpseudoephedrine

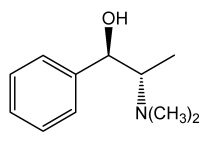

methylepherine
Fig. 5. Structures of 5 Ephedrine Alkaloids Used in Quantitative Analysis

Table 3. Primers of $18 \mathrm{~S}$ rRNA Gene and $\operatorname{trnK}$ Gene for PCR and Sequecing Reaction

\begin{tabular}{|c|c|c|}
\hline Primer & Sequence $\left(5^{\prime} \rightarrow 3^{\prime}\right)$ & Length (bp) \\
\hline \multicolumn{3}{|l|}{ 18S rRNA gene } \\
\hline \multicolumn{3}{|c|}{ Forward primer } \\
\hline $18 \mathrm{~S} 5^{\prime} \mathrm{F}^{a)}$ & CAA CCT GGT TGA TCC TGC CAG T & 22 \\
\hline FZ 418 & TCC AAG GAA GGC AGC AGG & 18 \\
\hline$\underline{18 \mathrm{~S} 891 \mathrm{~F}}$ & GTA TTT CAT TGT CAG AGG TG & 20 \\
\hline FZ 1308 & TTT GTC TGG TTA ATT CCG TT & 20 \\
\hline \multicolumn{3}{|c|}{ Reverse primer } \\
\hline RZ 376 & CCC TCT CCG GAA TCG AAC CC & 20 \\
\hline RZ 905 & ATC CAA GAA TTT CAC CTC T & 19 \\
\hline RZ 1310 & AAC GGA ATT AAC CAG ACA & 18 \\
\hline $\operatorname{ITS} 57 \mathrm{R}^{a)}$ & GAG AGC GAA CTT ACT CAG GGG TT & 23 \\
\hline
\end{tabular}

\begin{tabular}{|c|c|c|}
\hline \multicolumn{3}{|l|}{$\operatorname{trn} \mathrm{K}$ gene } \\
\hline \multicolumn{3}{|l|}{ Forward primer } \\
\hline $\operatorname{trnK} 3914 \mathrm{~F}^{a}$ & ()TGG GTT GCT AAC TCA ATG G & 19 \\
\hline $\operatorname{trnK}$ In $1 F$ & ATG ACG ATT GAG ATT CTT TC & 20 \\
\hline $\operatorname{trnK} \operatorname{In} 2 \mathrm{~F}^{a)}$ & AAT TTT CAA TCA GTC TTT TCT CTA & 24 \\
\hline $\operatorname{trnK}$ In $3 \mathrm{~F}$ & ATT GAT TTT GCT GGG AAC TAC TAT & 24 \\
\hline $\operatorname{trnK}$ In $4 \mathrm{~F}$ & GCA TGG CTT AGT TTT ACC GAC AAT & 24 \\
\hline \multicolumn{3}{|l|}{ Reverse primer } \\
\hline $\mathrm{nK}$ In $5 \mathrm{R}$ & CTT AGC AAC CAG GCG TTC TC & 20 \\
\hline $\operatorname{trnK}$ In 4R & AGA TAG TTT TGT TGA TGT AAT AGC & 24 \\
\hline $\operatorname{trnK} \operatorname{In} 3 \mathrm{R}^{a)}$ & CTT TAG CAA TCT TAT CGT GTC TTT & 24 \\
\hline $\operatorname{trnK}$ In $2 \mathrm{R}$ & ACG TTT ATT TAT TGC TTC ACT GTA & 24 \\
\hline $\operatorname{trnK}$ In 1R & TCA CAA AAA TTG ATC TAA CA & 20 \\
\hline $\operatorname{trnK} 2 \mathrm{R}^{a}$ & AAC TAG TCG GAT GGA GTA G & 19 \\
\hline
\end{tabular}

a) Primers for PCR amplification. New designed primers for Ephedra species are underlined.

Table 4. Nucleotide Differences in 18S rRNA Gene and trnK Gene of Ephedra Plants from China

Nucleotide position

\begin{tabular}{|c|c|c|c|c|c|c|c|c|c|c|c|c|c|c|c|c|c|c|c|}
\hline \multirow{5}{*}{ Species } & \multirow{5}{*}{ Genotype } & \multicolumn{4}{|c|}{ Nuclear 18S rRNA gene } & \multicolumn{14}{|c|}{ Chloroplast trnK gene } \\
\hline & & & 1 & 1 & 1 & & & & 6 & & & 1 & 1 & 1 & 1 & 2 & 2 & 2 & 2 \\
\hline & & 6 & 3 & 6 & 7 & 1 & 2 & 5 & $6-0$ & 7 & 8 & 0 & 1 & 8 & 9 & 1 & 1 & 1 & 1 \\
\hline & & 2 & 8 & 8 & 0 & 8 & 5 & 3 & 8 & 8 & 2 & 2 & 6 & 5 & 4 & 1 & 3 & 4 & 8 \\
\hline & & 5 & 1 & 3 & 9 & 2 & 0 & 6 & $(\text { units No.) })^{a)}$ & 7 & 5 & 7 & 7 & 7 & 6 & 5 & 7 & 4 & 5 \\
\hline Ephedra sinica & Si-II & A & $\mathrm{T}$ & $\mathrm{T}$ & $\mathrm{Y}$ & $\mathrm{T}$ & $\mathrm{C}$ & $\mathrm{C}$ & (1) & G & G & $\mathrm{C}$ & G & G & G & G & G & $\mathrm{C}$ & G \\
\hline E. intermedia & In & * & * & $*$ & $\mathrm{~T}$ & A & $*$ & $*$ & ( 2 or 3$)$ & $*$ & A & $*$ & $*$ & $*$ & $*$ & * & $*$ & * & * \\
\hline E. przewalskii & Pr-I & $*$ & * & $*$ & $\mathrm{C}$ & $\mathrm{A}$ & $*$ & $*$ & (1) & $*$ & $*$ & $*$ & $*$ & $*$ & $*$ & $*$ & $*$ & $*$ & * \\
\hline E. equisetina & E-I & G & $\mathrm{C}$ & $\mathrm{C}$ & $\mathrm{C}$ & $\mathrm{A}$ & G & $\mathrm{T}$ & (1) & $*$ & $*$ & $\mathrm{~T}$ & $*$ & A & $*$ & $\mathrm{~T}$ & $\mathrm{C}$ & $\mathrm{T}$ & A \\
\hline E. monosperma & E-I & G & $\mathrm{C}$ & $\mathrm{C}$ & $\mathrm{C}$ & A & $\mathrm{G}$ & $\mathrm{T}$ & (1) & $*$ & * & $\mathrm{T}$ & $*$ & A & $*$ & $\mathrm{~T}$ & $\mathrm{C}$ & $\mathrm{T}$ & A \\
\hline E. likiangensis & E-I & G & $\mathrm{C}$ & $\mathrm{C}$ & $\mathrm{C}$ & A & G & $\mathrm{T}$ & (1) & $*$ & * & $\mathrm{T}$ & $*$ & A & $*$ & $\mathrm{~T}$ & $\mathrm{C}$ & $\mathrm{T}$ & A \\
\hline E. gerardiana & E-I & G & $\mathrm{C}$ & $\mathrm{C}$ & $\mathrm{C}$ & A & $\mathrm{G}$ & $\mathrm{T}$ & (1) & $*$ & $*$ & $\mathrm{~T}$ & $*$ & A & $*$ & $\mathrm{~T}$ & $\mathrm{C}$ & $\mathrm{T}$ & A \\
\hline E. minuta & $\mathrm{Mi}$ & G & $\mathrm{C}$ & $\mathrm{C}$ & $\mathrm{C}$ & A & $\mathrm{G}$ & $\mathrm{T}$ & (1) & A & * & $\mathrm{T}$ & A & A & $\mathrm{C}$ & $\mathrm{T}$ & $*$ & $\mathrm{~T}$ & A \\
\hline
\end{tabular}

Numerals above sequence are nucleotide positions of $E$. sinica, which correspond to all other species except $E$. intermedia. Asterisk indicates the identical nucleotide to which of E. sinica. $\mathrm{Y}=\mathrm{C}$ and T. a) Sequence of a repeat unit is GTGTTATTTTATTTCTAAAAAGAAAGGTTTAGGAGGTT (38 bp). Two or three of this sequence unit have been observed in E. intermedia. 
Table 5. Nucleotide Differences in 18S rRNA Gene and trnK Gene of Ephedra Plants from Mongolia

\begin{tabular}{|c|c|c|c|c|c|c|c|c|c|c|c|c|c|c|c|c|c|c|c|c|c|c|}
\hline \multirow{6}{*}{ Species } & \multirow{6}{*}{ Genotype } & \multicolumn{21}{|c|}{ Nucleotide position } \\
\hline & & \multicolumn{4}{|c|}{$\begin{array}{c}\text { Nuclear } 18 \mathrm{~S} \text { rRNA } \\
\text { gene }\end{array}$} & \multicolumn{17}{|c|}{ Chloroplast $\operatorname{trn} \mathrm{K}$ gene } \\
\hline & & & 1 & 1 & 1 & & & & & & & & & 1 & 1 & 1 & 1 & 2 & 2 & 2 & 2 & 2 \\
\hline & & 6 & 3 & 6 & 7 & 1 & 2 & 5 & 7 & 8 & & 8 & 9 & 0 & 3 & 7 & 8 & 0 & 1 & 1 & 1 & 1 \\
\hline & & 5 & 8 & 8 & 0 & 8 & 5 & 3 & 5 & 8 & - & 8 & 9 & 2 & 2 & 8 & 5 & 7 & 1 & 3 & 4 & 8 \\
\hline & & 2 & 1 & 3 & 9 & 2 & 0 & 6 & 3 & 6 & & 8 & 8 & 7 & 0 & 5 & 7 & 1 & 5 & 7 & 4 & 5 \\
\hline \multirow[t]{2}{*}{ Ephedra sinica } & Si-I & $\mathrm{A}$ & $\mathrm{T}$ & $\mathrm{T}$ & $\mathrm{T}$ & $\mathrm{T}$ & $\mathrm{C}$ & $\mathrm{C}$ & $\mathrm{T}$ & & $\mathrm{G} \mathrm{T}$ & & $\mathrm{T}$ & $\mathrm{C}$ & $\mathrm{C}$ & $\mathrm{C}$ & $\mathrm{G}$ & $\mathrm{C}$ & $\mathrm{G}$ & $\mathrm{G}$ & $\mathrm{C}$ & G \\
\hline & Si-II & $*$ & $*$ & $*$ & $\mathrm{Y}$ & $*$ & $*$ & $*$ & $*$ & & $* *$ & $*$ & $*$ & $*$ & $*$ & $*$ & $*$ & $*$ & $*$ & $*$ & $*$ & * \\
\hline E. equisetina & E-I & $\mathrm{G}$ & $\mathrm{C}$ & $\mathrm{C}$ & $\mathrm{C}$ & $\mathrm{A}$ & G & $\mathrm{T}$ & $*$ & & $* *$ & $*$ & $*$ & $\mathrm{~T}$ & $*$ & $*$ & $\mathrm{~A}$ & $*$ & $\mathrm{~T}$ & $\mathrm{C}$ & $\mathrm{T}$ & A \\
\hline E. monosperma & E-I & $\mathrm{G}$ & $\mathrm{C}$ & $\mathrm{C}$ & $\mathrm{C}$ & $\mathrm{A}$ & G & $\mathrm{T}$ & $*$ & & $* *$ & $*$ & $*$ & $\mathrm{~T}$ & $*$ & $*$ & A & $*$ & $\mathrm{~T}$ & $\mathrm{C}$ & $\mathrm{T}$ & A \\
\hline \multirow[t]{3}{*}{ E. przewalskii } & Pr-I & $*$ & $*$ & $*$ & $\mathrm{C}$ & A & $*$ & $*$ & $*$ & & $* *$ & $*$ & $*$ & $*$ & $*$ & $*$ & $*$ & $*$ & $*$ & $*$ & $*$ & $*$ \\
\hline & Pr-II & $*$ & * & $*$ & $\mathrm{C}$ & $\mathrm{A}$ & $*$ & $*$ & $\mathrm{C}$ & & $* *$ & $*$ & $*$ & $*$ & $*$ & $*$ & $*$ & $*$ & $*$ & $*$ & $*$ & $*$ \\
\hline & Pr-III & $*$ & $*$ & $*$ & $\mathrm{C}$ & $\mathrm{A}$ & $*$ & $*$ & $*$ & & $* *$ & $*$ & $*$ & $*$ & $\mathrm{~T}$ & $*$ & $*$ & $*$ & $*$ & $*$ & $*$ & * \\
\hline \multirow[t]{2}{*}{ E. regeliana } & Rg-I & $*$ & $*$ & $*$ & $\mathrm{C}$ & $\mathrm{A}$ & $*$ & $*$ & $*$ & & $\mathrm{CA}$ & & $\mathrm{C}$ & $*$ & $*$ & $\mathrm{~T}$ & A & $*$ & $*$ & $*$ & * & * \\
\hline & Rg-II & $*$ & $*$ & $*$ & $\mathrm{C}$ & A & $*$ & $*$ & $*$ & & $\mathrm{CA}$ & & $\mathrm{C}$ & $*$ & $*$ & $\mathrm{~T}$ & A & $\mathrm{T}$ & $*$ & $*$ & $*$ & * \\
\hline Ephedra sp. & Esp & $*$ & $*$ & $*$ & $\mathrm{Y}$ & $\mathrm{A}$ & $*$ & $*$ & $*$ & & $* *$ & $*$ & $*$ & $*$ & $*$ & $*$ & $*$ & $*$ & $*$ & $*$ & $*$ & * \\
\hline
\end{tabular}
and T.

Numerals above sequence are nucleotide positions of $E$. sinica, which correspond to all other species. Asterisk indicates the identical nucleotide to which of $E$. sinica . $\mathrm{Y}=\mathrm{C}$

lian Ephedra plants into 9 genotypes (Table 5). E. sinica, E. equisetina and E. monosperma presented completely identical sequences in both gene regions to those of corresponding species from China (genotypes Si-II and E-I). However, two samples of E. sinica collected at points E11 and E28 showed a single peak of $\mathrm{T}$ (genotype Si-I) at position 1709 in $18 \mathrm{~S}$ rRNA gene sequence, differing from others which showed obviously double peaks $(\mathrm{T}>\mathrm{C})$ as that of Chinese ones except one sample (M1069) with almost equal intensity of T and $\mathrm{C}(\mathrm{T} \fallingdotseq \mathrm{C})$. E. przewalskii was of the same $18 \mathrm{~S}$ rRNA gene sequence as the Chinese congener, whereas three types of $\operatorname{trnK}$ gene sequences were observed. One type was the same as that of the same species from China (genotype Pr-I) and the other two differed from Pr-I type at 1 nucleotide site (genotypes Pr-II and Pr-III). E. regeliana collected in western Mongolia around Hyargas Lake, possessed its own specific nucleotide sequence, differing considerably from other Mongolian species at $1-4$ nucleotide sites in 18S rRNA gene and at $6-13$ sites in $t r n \mathrm{~K}$ gene region. The unknown ESP had identical sequence to genotype Si-II in the 18S rRNA gene region and to genotype Pr-I in the trnK gene region, and so was assigned as genotype Esp. The relative intensities of $\mathrm{C}$ and $\mathrm{T}$ at position 1709 in 18S rRNA gene sequence varied among samples, approximately two-thirds of the samples were $\mathrm{T}>\mathrm{C}$ and one-third were $\mathrm{C}>\mathrm{T}$ or $\mathrm{T} \fallingdotseq \mathrm{C}$.

Contents of Ephedrine Alkaloids in Mongolian Samples In the HPLC chromatograms, the five ephedrine alkaloids were detected sequentially as norpseudoephedrine (retention time, $25.0 \pm 0.8 \mathrm{~min})$, norephedrine $(26.8 \pm 0.8 \mathrm{~min})$, pseudoephedrine $(28.5 \pm 0.9 \mathrm{~min})$, ephedrine $(31.4 \pm 0.9 \mathrm{~min})$ and methylephedrine $(33.8 \pm 1.0 \mathrm{~min})$. Contents of the five ephedrine alkaloids in the stem of Mongolian Ephedra plants were quantitatively analyzed (Table 6).

Except for E. przewalskii (genotypes Pr-I, Pr-II and Pr-III), all samples contained high amounts of total ephedrine alkaloids (TAs), ranging from 1.86 to $4.90 \%$ of dry weight (Table 6). In regard to the relative contents of the two main constituents, ephedrine (Eph) and pseudoepedrine (PEph), a high percentage of PEph in TAs was observed in Mongolian
Ephedra plants, differing from the Chinese. ${ }^{22)}$

E. sinica (genotypes Si-I and Si-II) contained 1.95$4.16 \%$ TAs and showed a relatively higher percentage of Eph in TAs $(10.6-77.8 \%)$ than other species ( $\leqq 35.8 \%)$. Within the species, the samples collected from eastern grassland areas showed quite a bit higher content of Eph $(33.2-77.8 \%$ of TAs) than those from central areas (10.6- $47.3 \%$ of TAs, Table 6, Fig. 6). E. equisetina showed the highest content of TAs (3.98-4.90\%) and more than $90 \%$ of TAs was PEph. The two species satisfied the criterion in Japanese Pharmacopoeia that Eph and PEph comprise no less than $0.7 \%$ of the total: the sum contents were $1.43-3.68 \%$ in E. sinica and $3.81-4.59 \%$ in E. equisetina (Table 6). Therefore, these two species growing in Mongolia were found applicable as a new resource of "Mao" for Kampo medicine.

E. regeliana contained a similar amount of TAs as $E$. sinica, but the content of PEph accounted for more than $70 \%$ of TAs. Caveney et al. ${ }^{2)}$ reported that this species collected in Karakoram, Pakistan contained a comparable amount of ephedrine alkaloids to E. intermedia, but Long et al. ${ }^{16)}$ did not detect ephedrine alkaloids in their samples of this species collected in Xinjiang, China. These differences might be attributable to the growth environments or to taxonomic divergence in this species.

ESP (genotype Esp) showed higher TAs content (2.064.78\%) and higher percentage of PEph in TAs (45.0-95.7\% of TAs) than E. sinica. In addition, some samples presented allopheric features as shown in Fig. 2. The left part showed general morphology of ESP and the right part was similar to E. przewalskii. We analyzed separately the ESP parts and the E. przewalskii-resembling parts to check whether there were any differences in alkaloid content between these two parts. The results showed that generally the ESP part had higher ephedrine alkaloid content, but the relative compositions of the five alkaloids were similar.

\section{DISCUSSION}

Mongolia is one of the main habitats of Ephedra plants. 
Table 6. Ephedrine Alkaloids Content of Mongolian Ephedra Plants

\begin{tabular}{|c|c|c|c|c|c|c|c|c|c|c|c|c|c|}
\hline \multirow{2}{*}{ Point } & \multirow{2}{*}{ Species } & \multirow{2}{*}{ Genotype } & \multirow{2}{*}{ Voucher No. } & & \multicolumn{5}{|c|}{ Contents (\%) } & \multirow{2}{*}{$\begin{array}{l}\text { TAs } \\
\text { content } \\
(\%)\end{array}$} & \multirow{2}{*}{$\begin{array}{l}\text { Indication } \\
\text { content in } \\
\mathrm{JP}(\%)^{a)}\end{array}$} & \multirow{2}{*}{$\begin{array}{l}\text { Eph/ } \\
\text { TAs } \\
(\%)\end{array}$} & \multirow{2}{*}{$\begin{array}{c}\text { PEph/ } \\
\text { TAs } \\
(\%)\end{array}$} \\
\hline & & & & & Eph & PEph & NEph & NPEph & MEph & & & & \\
\hline E28 & Ephedra sinica & Si-I & M1157 & & 0.99 & 0.84 & 0.15 & 0.29 & $\operatorname{tr}$ & 2.26 & 1.82 & 43.7 & 37.1 \\
\hline E16 & Ephedra sinica & Si-II & M969 & & 0.39 & 2.24 & 0.33 & 0.69 & $\operatorname{tr}$ & 3.65 & 2.63 & 10.6 & 61.4 \\
\hline E17 & Ephedra sinica & $\mathrm{Si}$-II & M990 & & 0.52 & 1.57 & $\operatorname{tr}$ & 0.15 & 0.12 & 2.36 & 2.10 & 22.1 & 66.8 \\
\hline E24 & Ephedra sinica & Si-II & M1054 & & 0.64 & 0.97 & 0.17 & 0.13 & 0.07 & 1.98 & 1.61 & 32.2 & 48.9 \\
\hline E24 & Ephedra sinica & Si-II & M1056 & & 1.97 & 1.66 & 0.22 & 0.11 & 0.20 & 4.16 & 3.63 & 47.3 & 39.9 \\
\hline E25 & Ephedra sinica & Si-II & M1069 & & 1.04 & 1.83 & 0.10 & 0.42 & 0.14 & 3.53 & 2.87 & 29.5 & 51.8 \\
\hline E26 & Ephedra sinica & Si-II & M1071 & & 0.38 & 1.62 & 0.18 & 0.25 & 0.10 & 2.53 & 2.00 & 15.0 & 64.0 \\
\hline E27 & Ephedra sinica & Si-II & M1156 & & 1.35 & 1.31 & 0.09 & 0.25 & 0.20 & 3.20 & 2.66 & 42.2 & 40.9 \\
\hline E29 & Ephedra sinica & Si-II & M1210 & & 0.90 & 1.45 & $\operatorname{tr}$ & 0.14 & 0.22 & 2.71 & 2.35 & 33.2 & 53.5 \\
\hline E30 & Ephedra sinica & Si-II & M1211 & & 2.14 & 0.22 & $\operatorname{tr}$ & 0.07 & 0.32 & 2.75 & 2.36 & 77.8 & 8.0 \\
\hline E31 & Ephedra sinica & Si-II & M1213 & & 1.28 & 1.12 & 0.07 & 0.26 & 0.19 & 2.92 & 2.40 & 43.9 & 38.4 \\
\hline E32 & Ephedra sinica & Si-II & M1215 & & 1.15 & 0.47 & $\operatorname{tr}$ & 0.12 & 0.12 & 1.86 & 1.62 & 61.8 & 25.1 \\
\hline E33 & Ephedra sinica & Si-II & M1227 & & 1.64 & 1.17 & 0.34 & 0.73 & 0.12 & 4.00 & 2.81 & 41.0 & 29.3 \\
\hline E34 & Ephedra sinica & Si-II & M1229 & & 1.00 & 0.88 & $\operatorname{tr}$ & 0.25 & 0.11 & 2.24 & 1.88 & 44.7 & 39.2 \\
\hline E35 & Ephedra sinica & Si-II & M1242 & & 1.13 & 0.30 & 0.14 & 0.27 & 0.11 & 1.95 & 1.43 & 57.9 & 15.4 \\
\hline E18 & Ephedra equisetina & E-I & M995 & & 0.14 & 4.45 & 0.12 & 0.12 & 0.07 & 4.90 & 4.59 & 2.8 & 90.8 \\
\hline E18 & Ephedra equisetina & E-I & M998 & & 0.15 & 3.66 & $\operatorname{tr}$ & 0.07 & 0.10 & 3.98 & 3.81 & 3.7 & 91.9 \\
\hline E10 & Ephedra przewalskii & Pr-II & M871 & & - & $\operatorname{tr}$ & - & - & $\operatorname{tr}$ & $\operatorname{tr}$ & $\operatorname{tr}$ & - & - \\
\hline E14 & Ephedra przewalskii & Pr-III & M931 & & $\operatorname{tr}$ & $\operatorname{tr}$ & $\operatorname{tr}$ & - & $\operatorname{tr}$ & $\operatorname{tr}$ & $\operatorname{tr}$ & - & - \\
\hline E20 & Ephedra przewalskii & Pr-III & M1028 & & - & $\operatorname{tr}$ & - & - & - & $\operatorname{tr}$ & $\operatorname{tr}$ & - & - \\
\hline $\mathrm{E} 42$ & Ephedra przewalskii & Pr-I & M1348-1 & & $\operatorname{tr}$ & $\operatorname{tr}$ & $\operatorname{tr}$ & - & - & $\operatorname{tr}$ & $\operatorname{tr}$ & - & - \\
\hline E42 & Ephedra przewalskii & Pr-I & M1348-2 & & $\operatorname{tr}$ & $\operatorname{tr}$ & $\operatorname{tr}$ & - & - & $\operatorname{tr}$ & $\operatorname{tr}$ & - & - \\
\hline E6 & Ephedra regeliana & Rg-II & M779 & & 0.41 & 1.44 & $\operatorname{tr}$ & $\operatorname{tr}$ & 0.10 & 1.95 & 1.85 & 21.1 & 74.0 \\
\hline E7 & Ephedra regeliana & Rg-I & M1301 & & $\operatorname{tr}$ & 2.78 & $\operatorname{tr}$ & 0.35 & $\operatorname{tr}$ & 3.13 & 2.78 & - & 88.8 \\
\hline E7 & Ephedra regeliana & Rg-I & M1302 & & 0.59 & 2.25 & 0.13 & 0.14 & 0.08 & 3.19 & 2.84 & 18.5 & 70.6 \\
\hline E13 & Ephedra sp. & Esp & M928-1 & & 0.48 & 2.87 & 0.25 & 0.66 & $\operatorname{tr}$ & 4.27 & 3.35 & 11.2 & 67.3 \\
\hline E13 & Ephedra sp. & Esp & M928-2 & & 0.53 & 3.15 & 0.31 & 0.79 & $\operatorname{tr}$ & 4.77 & 3.68 & 11.1 & 66.0 \\
\hline E13 & Ephedra sp. & Esp & M928-3 & & 0.41 & 3.10 & 0.25 & 0.76 & $\operatorname{tr}$ & 4.52 & 3.51 & 9.0 & 68.6 \\
\hline E15 & Ephedra sp. & Esp & M950 & & 1.01 & 3.19 & $\operatorname{tr}$ & 0.20 & 0.18 & 4.58 & 4.20 & 22.1 & 69.5 \\
\hline E15 & Ephedra sp. & Esp & M951 & & 0.14 & 2.73 & $\operatorname{tr}$ & 0.10 & $\operatorname{tr}$ & 2.97 & 2.87 & 4.6 & 91.9 \\
\hline E19 & Ephedra sp. & Esp & M1027-1 & & 1.13 & 2.58 & 0.18 & 0.74 & $\operatorname{tr}$ & 4.61 & 3.70 & 24.4 & 55.9 \\
\hline E19 & Ephedra sp. & Esp & M1027-2 & & 0.59 & 1.67 & 0.63 & 0.82 & $\operatorname{tr}$ & 3.72 & 2.27 & 15.9 & 45.0 \\
\hline E20 & Ephedra sp. & Esp & M1029 & & 0.37 & 3.00 & $\operatorname{tr}$ & 0.23 & $\operatorname{tr}$ & 3.60 & 3.37 & 10.3 & 83.4 \\
\hline E22 & Ephedra sp. & Esp & M1036 & & 1.03 & 1.98 & $\operatorname{tr}$ & 0.34 & 0.08 & 3.43 & 3.01 & 30.0 & 57.8 \\
\hline E22 & Ephedra sp. & Esp & M1037 & & 0.83 & 2.08 & $\operatorname{tr}$ & 0.37 & $\operatorname{tr}$ & 3.28 & 2.91 & 25.2 & 63.5 \\
\hline E22 & Ephedra sp. & Esp & M1039 & & 0.35 & 1.28 & 0.18 & 0.26 & $\operatorname{tr}$ & 2.06 & 1.62 & 16.8 & 62.0 \\
\hline E23 & Ephedra sp. & Esp & M1051 & & 1.31 & 1.71 & $\operatorname{tr}$ & 0.51 & 0.13 & 3.66 & 3.02 & 35.8 & 46.8 \\
\hline E23 & Ephedra sp. & Esp & M1052 & & 0.41 & 2.52 & $\operatorname{tr}$ & 0.07 & 0.16 & 3.16 & 2.93 & 13.0 & 79.6 \\
\hline \multirow[t]{2}{*}{ E13 } & Ephedra sp. & Esp & M929 & $* *$ & 0.68 & 3.16 & $\operatorname{tr}$ & 0.17 & 0.16 & 4.16 & 3.83 & 16.3 & 75.8 \\
\hline & & & & $* * *$ & 0.42 & 1.90 & $\operatorname{tr}$ & 0.19 & 0.08 & 2.52 & 2.33 & 16.8 & 75.6 \\
\hline \multirow[t]{2}{*}{ E13 } & Ephedra sp. & Esp & M930 & $* *$ & 0.77 & 3.49 & $\operatorname{tr}$ & 0.19 & 0.17 & 4.61 & 4.26 & 16.7 & 75.7 \\
\hline & & & & $* * *$ & 0.59 & 2.14 & $\operatorname{tr}$ & 0.27 & 0.09 & 3.00 & 2.73 & 19.6 & 71.4 \\
\hline \multirow[t]{2}{*}{ E15 } & Ephedra sp. & Esp & M952 & $* *$ & 1.06 & 3.36 & $\operatorname{tr}$ & 0.19 & 0.16 & 4.78 & 4.42 & 22.2 & 70.4 \\
\hline & & & & $* * *$ & 0.73 & 2.20 & $\operatorname{tr}$ & 0.17 & 0.10 & 3.10 & 2.93 & 23.5 & 71.1 \\
\hline \multirow[t]{2}{*}{ E15 } & Ephedra sp. & Esp & M953 & $* *$ & 0.43 & 3.40 & $\operatorname{tr}$ & 0.13 & 0.12 & 4.09 & 3.83 & 10.5 & 83.3 \\
\hline & & & & $* * *$ & 0.88 & 1.84 & $\operatorname{tr}$ & 0.24 & 0.09 & 2.95 & 2.71 & 29.7 & 62.3 \\
\hline \multirow[t]{2}{*}{ E15 } & Ephedra sp. & Esp & M954 & $* *$ & 0.79 & 2.68 & $\operatorname{tr}$ & 0.11 & 0.16 & 3.74 & 3.47 & 21.2 & 71.7 \\
\hline & & & & $* * *$ & 0.52 & 2.32 & $\operatorname{tr}$ & 0.08 & 0.12 & 2.91 & 2.83 & 17.7 & 79.5 \\
\hline \multirow[t]{2}{*}{ E21 } & Ephedra sp. & Esp & M1030 & $* *$ & $\operatorname{tr}$ & 3.00 & $\operatorname{tr}$ & 0.23 & $\operatorname{tr}$ & 3.23 & 3.00 & - & 93.0 \\
\hline & & & & $* * *$ & $\operatorname{tr}$ & 2.30 & $\operatorname{tr}$ & 0.10 & $\operatorname{tr}$ & 2.40 & 2.30 & - & 95.7 \\
\hline
\end{tabular}

Eph: ephedrine, PEph: pseudoephedrine, NEph: norephedrine, NPEph: norpseudoephedrine, MEph: methylephedrine. Indication content in JP: sum contents of Eph and PEph. TAs content: total content of Eph, PEph, NEph, NPEph and MEph. a) JP: Japanese Pharmacopoeia, tr: $<0.07 \%$, : not detected. Sample with allopheric features was analyzed separately and contents of ESP part (**) and E. przewalskii-resembling part (***) are shown.

Nine species are reported to distribute throughout the country. The characters of seed, integument tube, bracts of seed cone, etc. were particularly important for morphological identification of these plants. ${ }^{10-12,19,20)}$ We made great efforts to conduct field investigations in summer, the season of blossoming and fruiting of Ephedra plants. But in many habitats, reproductive organs had not been observed, which made morphological identification difficult. Moreover, it has been pointed out that even the cut products of Ephedrae herba were impossible to be identified by morphological features.
The present study proposed genetic markers for objective authentication of Ephedra plants and Ephedrae herba. Although intra-species variation was observed in E. sinica, E. przewalskii and E. regeliana, the five species and ESP collected in Mongolia could be discriminated from each other by the nucleotide sequences of the $18 \mathrm{~S}$ rRNA-trnK genes. The distribution of Ephedra plants in Mongolia and their genotypes is shown in Fig. 1, integrating the results from field investigations and molecular analysis. E. sinica (genotypes Si-I and Si-II) was found in wide areas of eastern and central areas; $E$. 


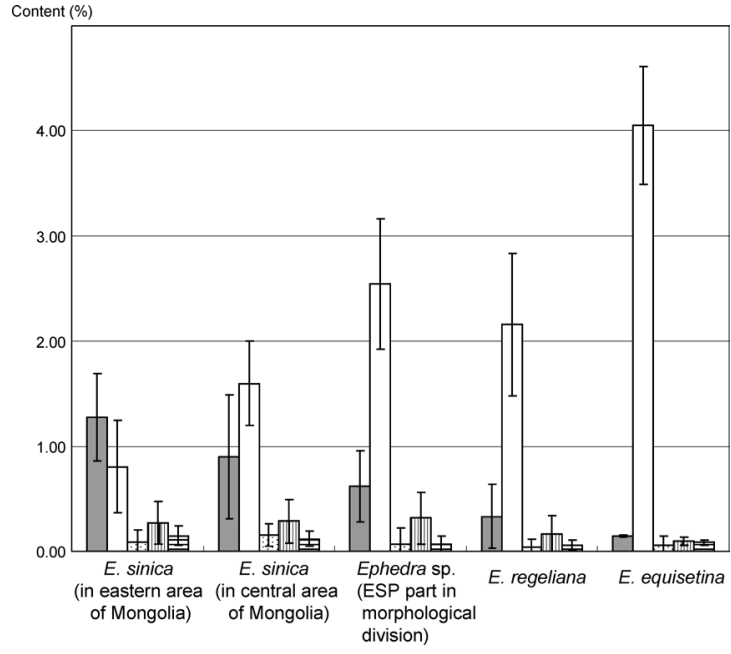

Fig. 6. Contents of Five Ephedrine Alkaloids in Mongolian Ephedra Species

Shaded column, ephedrine; white column, pseudoephedrine; sandy column, norephedrine; vertical line column, norpseudoephedrine; horizontal line column, methelephedrine. Contents are indicated as mean \pm S.D.

equisetina was found in the mountainous region of the southcentral area; E. przewalskii with genotypes Pr-I and Pr-III was found mainly in the southern area, growing together with Ephedra sp. (genotype Esp), and those with genotype Pr-II were at Sharga in the western area; E. monosperma was growing in a high mountain area (alt. $1845 \mathrm{~m}$ ) near Hovsgol Lake in the northern area and E. regeliana (genotypes Rg-I and $\mathrm{Rg}$-II) was found on western rocky slopes.

According to conventional morphological taxonomy, $E$. przewalskii is well separated from other Ephedra species by the texture of its cone bracts in mature seed. ${ }^{19)}$ E. przewalskii belongs to Section Alatae which is characterized by dry and winged bracts in mature seed, whereas others belong to Section Ephedra which possesses fleshy cone bracts. However, the present molecular data of $18 \mathrm{~S}$ rRNA-trnK gene sequences did not support this classification, and E. przewalskii was genetically close to E. sinica and E. intermedia. Previous phylogenetic studies on Ephedra plants from China, EuropeMediterranean, and America based on nucleotide sequences of ITS1, ITS2, trnL/trnF, matK and $r b c \mathrm{~L}$ regions also suggest that the traditional classification on the basis of bracts texture might be unnatural. ${ }^{15,23)}$

Although at first glance ESP was similar to $E$. sinica distributed in eastern and central areas, the characteristic features such as a height less than $25 \mathrm{~cm}$, a stem often procumbent and with curved or twisted apex, etc. were different from $E$. sinica. These characters were clearly mentioned as discriminating $E$. sinica from $E$. distachya in "Flora of China"10) and "Flora Xingiangensis"19); that is, the morphology of ESP was thought to be consistent with E. distachya L. On the other hand, in "Flora of Siberia," E. dahurica Turz. was also described as having a curved or twisted stem, and it was treated as a synonym of $E$. distachya auct. non L. ${ }^{20)}$ In "Flora of Mongolia,"11) E. dahurica and E. sinica are listed separately, but $E$. distachya is not included, whereas in "Flora of China" and "Flora Xingiangensis," there is no E. dahurica citation. Due to these inconsistencies, further detailed investigation is needed to clarify the relation among the three species. In the present study genetic data suggested a close relation of ESP to E. sinica. Some specimens of genotype Esp revealed allopheric features in the same plant. As shown in Fig. 2, the left part of the specimen shows general features of ESP, but the right part resembles E. przewalskii in color and stem roughness, number of scaly leaves, etc. We assumed that the very different features of these two parts might have resulted from growth periods, that is, the ESP part was the newer portion, younger than the E. przewalskiiresembling part. Higher ephedrine content of ephedrine alkaloids in the ESP parts was detected, which might be indirectly explained by the observation of Malla et al., ${ }^{24)}$ who found by tracing alkaloid content during 9 years of cultivation that ephedrine alkaloid in E. gerardiana decreased after 4 years. On the other hand, the nuclear rDNA sequence which is of biparental inheritance has been demonstrated to be effective in speculation of hybridization. ${ }^{25)}$ The $18 \mathrm{~S}$ rRNA gene sequence of the samples of allopheric features presented 1 site of double peaks (C and $\mathrm{T}$ ) at position 1709 where high intensity of $\mathrm{C}(\mathrm{C}>\mathrm{T}$ or $\mathrm{C} \fallingdotseq \mathrm{T})$ differed from others, which possibly suggested a hybrid origin of these samples. Considering these genetic data (genotype Esp consisting of Si-II type 18S rRNA gene sequence and Pr-I type trnK gene sequence) as well as the distribution areas, the derivation of the allopheric specimens might be related to the pure line of ESP or its relatives and E. przewalskii. However, 18S rRNA gene presented hundreds or thousands of tandem repeats in the genome, and additive peaks at position 1709 resulting from one site of nucleotide difference among these repeated copies could not be absolutely excluded. Further studies, such as determination of other nuclear DNA regions as internal transcribed spacer or 5S rRNA spacer region together with detailed field investigations and even a crossing experiment, are needed to resolve the taxonomic position of these samples.

Almost all Mongolian Ephedra plants (except E. przewalskii) contained high amounts of TAs, $1.86-4.90 \%$ and a high percentage of pseudoephedrine in TAs (except $E$. sinica growing in the eastern area). On the other hand, it was reported that Chinese Ephedra plants contained more Eph than PEph, except for E. intermedia. ${ }^{22)}$ The phenomenon that the content ratio of Eph to PEph varied considerably even in the same species was much attractive from the viewpoint of biosynthetic regulation of these two constituents. Further investigation of the regulatory mechanism on formation of these two compounds is ongoing. PEph was reported to have effect of anti-tussive, anti-inflammation and other effects, ${ }^{4,26)}$ suggesting the usefulness of Mongolian Ephedra plants to relieve rhinitis and nasal congestion.

In conclusion, through field investigations together with subsequent laboratory research, the present study assessed the molecular and chemical diversity of Ephedra plants in Mongolia. The determined nucleotide sequences of $18 \mathrm{~S}$ rRNA gene and $\operatorname{trn\mathrm {K}}$ gene provided a useful index for identification and taxonomic classification. Chemical assessment suggested that $E$. sinica and E. equisetina found in eastern and central Mongolia had potential as new resources of Ephedrae herba of Japanese Pharmacopoeia grade and that other species could be resources of ephedrine alkaloids. Except for E. przewalskii, other species were characterized by a relatively high ratio of pseudoephedrine. Moreover, the deterioration of the natural environment, especially the progress 
of desertification in the southern area of Mongolia has been serious in recent years. It is necessary to promote the cultivation of selected species in selected suitable regions to secure a resource for Ephedrae herba and also for environmental preservation. Our results are believed to contribute much basic data to this effort.

Acknowledgments This work was supported by Grantsin-Aid for Scientific Research (B), No. 14406030 in 2002 2004 and No. 17406004 in 2005-2007 from the Japan Society for the Promotion of Science, and by a grant for Expansion Program, Knowledge Cluster (Hokuriku Innovation Cluster for Health Science) from the Ministry of Education, Culture, Sports, Science and Technology of Japan. The authors thank Dr. Yukiko Makino, Kanto-Shinetsu Regional Bureau of Health and Welfare for kindly providing norephedrine.

\section{REFERENCES}

1) Namba T., "The Encyclopedia of Wakan-Yaku (Traditional SinoJapanese Medicines) with Color Pictures," New Completely Revised Ed., Vol. II, Hoikusha, Osaka, 1994, pp. 53-57.

2) Caveney S., Charlet D. A., Freitag H., Maier-Stole M., Starratt A. N., Am. J. Bot., 88, 1199-1208 (2001).

3) Tanaka C., Kato R., "New Pharmacology," 6th ed., Nankodo, Tokyo, 2007 , pp. $266-267$.

4) Minamizawa K., Goto H., Ohi Y., Shimada Y., Terasawa K., Haji A., J. Pharmacol. Sci., 102, 136-142 (2006).

5) Carlini E. A., Pharmacol. Biochem. Behav., 75, 501-512 (2003).

6) Sandeep S. V., Dennis R. F., Biochem. Pharmacol., 58, 807-810 (1999).

7) U. S. Food and Drug Administration, 〈http://www.fda.gov/oc/initiatives/ephedra/february2004), FDA Web.

8) "Relative Information on Medicinal Plants (Crude Drugs)," Japan Spe- ciality Agriculture Products Association, Tokyo, 2004, p. 93.

9) State Food and Drug Administration, 〈http://www.gddoftec.gov.cn/ wjmzc_080107/Detail.asp?ID $=1850\rangle$, Department of Foreign Trade and Economic Cooperation of Guangdong Province Web.

10) Wu Z. Y., Peter H. R., "Flora of China," Vol. 4, Missouri Botanical Garden Press, St. Louis, 1999, pp. 97-101.

11) Grubov I. A., "Flora of Mongolia," Balanga, Moscow, 1996, p. 14.

12) Grubov V. I., "Key to the Vascular Plants of Mongolia (with an Atlas)," Vol. 1, Science Publishers, Inc., New Hampshire, 2001, pp. $30-32,272$.

13) Schaneberg B. T., Crockett S., Bedir E., Khan, I. A., Phytochemistry, 62, $911-918$ (2003).

14) Guo Y., Tsuruga A., Yamaguchi S., Oba K., Iwai K., Sekita S., Mizukami H., Biol. Pharm. Bull., 29, 1207-1211 (2006).

15) Long C. F., Kakiuchi N., Takahashi A., Komatsu K., Cai S. Q., Mikage M., Planta Med., 70, 1080-1084 (2004).

16) Long C. F., Kakiuchi N., Zhong G. Y., Mikage M., Biol. Pharm. Bull., 28, 285-288 (2005).

17) Zhu S., Fushimi H., Cai S. Q., Komatsu K., Planta Med., 69, 647653 (2003)

18) Cao H., Sasaki Y., Fushimi H., Komatsu K., Biol. Pharm. Bull., 24 1389-1394 (2001).

19) Yang C. Y., Shen K. M., Mao Z. M., "Flora Xinjiangensis," Tomus 1, Xinjiang Science \& Technology \& Hygiene Publishing House, Wulumuqi, 1992, pp. 87-109.

20) Krasnoborov I. M., "Flora of Siberia," Vol. 1, Science Publishers, New Hampshire, 2000, pp. 74, 76-77, 151-152.

21) Kondo N., Mikage M., Idaka K., Nat. Med., 53, 194-200 (1999).

22) Zhang J. S., Tian Z., Lou Z. C., Acta Pharmaceutica Sinica, 24, 865871 (1989).

23) Huang J., Giannase D. E., Price R. A., Mol. Phylogenet. Evol., 35, 48-59 (2005).

24) Malla K. J., Mitsuhashi A., Miyajima T., Watanabe T., Kitajima J., Arai Y., Takano A., Nat. Med., 59, 237-240 (2005).

25) Rauscher J. T., Doyle J. J., Brown A. H., Mol. Ecol., 11, 2691-2702 (2002).

26) Hikino H., Konno C., Takata H., Tamada M., Chem. Pharm. Bull., 28, 2900-2904 (1980). 